\title{
Utility of ultra-sensitive qPCR to detect Plasmodium falciparum and Plasmodium vivax infections under different transmission intensities
}

Maria Gruenberg 1,2, Clara Antunes Moniz 1,2, Natalie E. Hofmann 1,2, Cristian Koepfli 3,12, Leanne J. Robinson 4,13, Elma Nate ${ }^{4}$, Wuelton Marcelo Monteiro ${ }^{5}$, Gisely Cardoso de Melo ${ }^{5}$, Andrea Kuehn ${ }^{5,8}$, Andre M. Siqueira ${ }^{5,6}$, Wang Nguitragool ${ }^{7}$, Quique Bassat ${ }^{8}$, Marcus Lacerda ${ }^{5,9}$, Jetsumon Sattabongkot ${ }^{10}$, Ivo Mueller $3,11,14$ and Ingrid Felger ${ }^{1,2^{*}}$ (D)

\begin{abstract}
Background: The use of molecular diagnostics has revealed an unexpectedly large number of asymptomatic low-density malaria infections in many malaria endemic areas. This study compared the gains in parasite prevalence obtained by the use of ultra-sensitive (us)-qPCR as compared to standard qPCR in cross-sectional surveys conducted in Thailand, Brazil and Papua New Guinea (PNG). The compared assays differed in the copy number of qPCR targets in the parasite genome.
\end{abstract}

Methods: Plasmodium falciparum $(P f)$ and Plasmodium vivax $(P v)$ parasites were quantified by $q P C R$ amplifying the low-copy Pf_ and Pv_18S rRNA genes or the multi-copy targets Pf_varATS and Pv_mtCOX1. Cross-sectional surveys at the three study sites included 2252 participants of all ages and represented different transmission intensities.

Results: In the two low-transmission areas, P. falciparum positivity was 1.3\% (10/773) (Thailand) and 0.8\% (5/651) (Brazil) using standard Pf_18S rRNA qPCR. In these two countries, P. falciparum positivity by Pf_varATS us-qPCR increased to $1.9 \%$ (15/773) and 1.7\% (11/651). In PNG, an area with moderate transmission intensity, P. falciparum positivity significantly increased from $8.6 \%(71 / 828)$ by standard qPCR to $12.2 \%(101 / 828)$ by us-qPCR. The proportions of $P$. falciparum infections not detected by standard qPCR were 33\%, 55\% and 30\% in Thailand, Brazil and PNG. Plasmodium vivax was the predominating species in Thailand and Brazil, with 3.9\% (30/773) and 4.9\% (32/651) positivity by PV_18S rRNA qPCR. In PNG, P. vivax positivity was similar to P. falciparum, at 8.0\% (66/828). Use of $P v \_m t C O X 1$ us-qPCR led to a significant increase in positivity to $5.1 \%(39 / 773), 6.4 \%(42 / 651)$ and $11.5 \%(95 / 828)$ in Thailand, Brazil, and PNG. The proportions of P. vivax infections missed by standard qPCR were similar at all three sites, with $23 \%, 24 \%$ and $31 \%$ in Thailand, Brazil and PNG.

Conclusion: The proportional gains in the detection of P. falciparum and P. vivax infections by ultra-sensitive diagnostic assays were substantial at all three study sites. Thus, us-qPCR yields more precise prevalence estimates for both $P$. falciparum and $P$. vivax at all studied levels of endemicity and represents a significant diagnostic improvement.

*Correspondence: ingrid.felger@unibas.ch

1 Swiss Tropical and Public Health Institute, Basel, Switzerland

Full list of author information is available at the end of the article

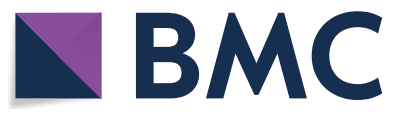

(c) The Author(s) 2020. This article is licensed under a Creative Commons Attribution 4.0 International License, which permits use, sharing, adaptation, distribution and reproduction in any medium or format, as long as you give appropriate credit to the original author(s) and the source, provide a link to the Creative Commons licence, and indicate if changes were made. The images or other third party material in this article are included in the article's Creative Commons licence, unless indicated otherwise in a credit line to the material. If material is not included in the article's Creative Commons licence and your intended use is not permitted by statutory regulation or exceeds the permitted use, you will need to obtain permission directly from the copyright holder. To view a copy of this licence, visit http://creativeco mmons.org/licenses/by/4.0/. The Creative Commons Public Domain Dedication waiver (http://creativecommons.org/publicdomain/ zero/1.0/) applies to the data made available in this article, unless otherwise stated in a credit line to the data. 
Improving sensitivity in $P$. vivax surveillance by us-qPCR is of particular benefit, because the additionally detected $P$. vivax infections signal the potential presence of hypnozoites and subsequent risk of relapse and further transmission.

Keywords: Ultra-sensitive, qPCR, Molecular diagnostics, Low-density, varATS, mtCOX1

\section{Background}

Asymptomatic Plasmodium infections, persisting undetected in the population, present a silent reservoir for ongoing transmission and pose a major challenge in malaria elimination [1]. Parasite densities in asymptomatic infections are often low, and therefore infections remain undetected by light microscopy (LM) or rapid diagnostic tests (RDT), the standard diagnostics to control malaria in endemic countries. In epidemiological studies, quantitative PCR (qPCR) has increasingly been used to obtain more precise prevalence rates for Plasmodium spp. infections [2-6]. The most widely used qPCR assays for detection of Plasmodium species target genus- or species-specific regions within the 18S rRNA genes [2, 7-9]. The genomes of Plasmodium falciparum $(P f)$ and $P$. vivax $(P v)$ harbour 5 and 3 copies of $18 \mathrm{~S}$ rRNA genes, however, some copies show substantial sequence variation so that primers and probes for P. vivax target only one copy of this gene family [10]. Comparison of the widely used $18 \mathrm{~S}$ rRNA assays with ultra-sensitive detection methods uncovered a 2-4 times higher parasite prevalence of lowdensity malaria infections at medium and low transmission settings that previously remained undiagnosed by less sensitive detection tools $[6,11,12]$.

Enhanced detection sensitivity by qPCR can be achieved in three ways: firstly, by processing larger volumes of blood and concentrating the DNA during the extraction procedure $[11,13]$, secondly, by using ultrasensitive-qPCR (us-qPCR) assays that target multi-copy sequences in the parasite genome $[10,11,14-16]$ instead of a single-copy or low-copy gene, and thirdly, by detecting highly abundant $18 \mathrm{~S}$ rRNA transcripts by quantitative reverse-transcriptase PCR (qRT-PCR) instead of rDNA. Collecting a large volume of blood by venipuncture instead of finger prick is mostly not feasible for large surveys. RNA should be preserved using specific buffers and stored at $-80{ }^{\circ} \mathrm{C}$ to avoid degradation. Compared to DNA-based tests, RNA extraction and qRT-PCR are more costly and the handling and amplification of the high copy $18 \mathrm{~S}$ rRNA transcripts are more prone to contamination $[2,10]$. Both these methods are, therefore, less practicable for malaria surveillance. In contrast, amplifying multi-copy genes by qPCR improves diagnostic sensitivity even when using finger prick blood samples [11]. For ultra-sensitive detection of low-density $P$. falciparum infections, the $P f_{-}$varATS us-qPCR was developed that targets the acidic terminal sequences of multi-copy var genes [11]. For low-density $P$. vivax infections, $P v_{-}$ mtCOX1 us-qPCR targeting the cytochrome oxidase 1 gene was designed [10]. COX1 is encoded in the mitochondrial genome and is present in at least 20 copies per cell [17]. Both us-qPCR assays for $P$. falciparum and $P$. vivax detect 10 -fold lower parasite densities as compared to $P f_{-}$and $P v_{-} 18 \mathrm{~S}$ rRNA assays $[10,11]$.

Ultra-sensitive diagnostic methods are of particular importance for $P$. vivax, as this species generally presents lower levels of parasitemia compared to $P$. falciparum due to its preferential invasion of the reticulocyte, rather than the mature red blood cell. For example in Papua New Guinea (PNG), where both species are equally prevalent, mean $P$. vivax parasite densities by LM in community samples were seven times lower than those of $P$. falciparum [3]. In the context of elimination, malaria diagnostics may require detection of the full extent of carriers of both parasite species. This is of particular relevance for $P$. vivax, as individuals with an undetected $P$. vivax infection may carry dormant hypnozoites and thus present a future reservoir for onward transmission due to later relapses.

Over the last decade, malaria epidemiological studies have increasingly employed molecular diagnostics, and PCR-based prevalence rates became available from many endemic areas $[1,16]$. Meta-analyses have investigated the relationship of PCR and LM prevalence for P. falciparum and $P$. vivax $[1,16,18]$. For $P$. falciparum this analysis has shown that the proportion of submicroscopic $P$. falciparum infections greatly varied according to $P$. falciparum transmission intensity and ranged from $20 \%$ in areas of high transmission to $80 \%$ in low endemicity settings [1]. For $P$. vivax, two systematic reviews analysed cross-sectional studies from Asia, South America and South Pacific and gave concordant results $[16,18]$, showing that PCR performed in community samples detected on average more than twice as many infections as LM. Similar to $P$. falciparum, the proportion of submicroscopic $P$. vivax infections was negatively correlated with prevalence by LM, i.e. a larger proportion of submicroscopic $P$. vivax infections was observed in low compared to high $P$. viva $x$ transmission areas [16].

Together these earlier studies indicated that in areas of low malaria endemicity, molecular diagnostic methods are of particular importance for assessing the transmission reservoir and for informing malaria control interventions $[1,16]$. The hypothesis for the current study 
was that us-qPCRs, compared to standard qPCRs, could also be particularly beneficial in low endemic areas, as they could further improve the precision of molecular prevalence estimates. The gain in additionally diagnosed parasite carriers by us-qPCR might also vary according to transmission intensity, similar to the described larger proportion of submicroscopic P. falciparum infections found in low endemic settings $[1,16]$. This study explored the advantage of using us-qPCR for $P$. falciparum and $P$. vivax compared to diagnosis by standard $18 \mathrm{~S}$ rRNA qPCR. By analysing community samples from low transmission settings in Brazil and Thailand and a setting of moderate transmission in PNG, the variation of gains across the study sites was examined, as well as the age distribution of submicroscopic infections by standard versus us-qPCR.

\section{Methods}

\section{Study sites and archived samples}

This study used 2252 archived DNA samples from crosssectional blood collections in Thailand, Brazil and PNG between 2012 and 2014. A detailed description of these three study sites and results by standard qPCR were published previously $[4,5,19]$ (W. Monteiro, pers. commun.). Sampling procedures, DNA extraction from red blood cell pellets and diagnostic tests were harmonized among all sites. DNA samples from participants of all age-groups were selected randomly from larger subsets from Thailand and Brazil. 773 archived DNA samples from Thailand originated from a low-endemic village of the BongTi region, Kanchanaburi province, and were collected in 2012. The 651 archived DNA samples from Brazil were collected in the Manaus region in March 2014. From PNG, the full sample sets (828 archived samples) from the two communities Megiar and Utu, one of high and one of low transmission, were analysed. These crosssectional surveys were conducted between May and July 2014 in Madang province. Prior to performance of
us-qPCR, good quality of the stored DNA was confirmed by repeating species-specific $P f_{-}$and $P v_{-} 18 \mathrm{~S}$ rRNA qPCR assays in a minimum of 150 samples from each study site. Results in these repeated subsets were compared to the first round of $P f_{-}$and $P v_{-} 18 \mathrm{~S}$ rRNA assays $[4,5,19]$. For Thailand and Brazil complete overlap for P. falciparumpositivity and almost complete overlap for $P$. vivax-positivity was found. For PNG P. falciparum-positivity agreed very well, but discrepancies were observed for $P$. vivax between first and repeat results. Therefore, all PNG samples were re-analysed by $P v_{-} 18 \mathrm{~S}$ rRNA qPCR and only the data from repeats were used for the comparison of diagnostic methods.

\section{qPCR and us-qPCR}

The three sets of samples were analysed in their respective countries of origin. For quantification of $P$. falciparum parasites in sample sets from Thailand and Brazil, a dilution of the WHO International Standard "Plasmodium falciparum DNA for NAT Assays" (NIBSC, UK) was used. For quantification of $P$. falciparum parasites in the PNG sample set, a serial dilution of cultured 3D7 parasites was used. Plasmodium vivax samples were quantified using a standard curve generated from dilutions of $P$. vivax control plasmids containing inserts corresponding to the respective qPCR or us-qPCR target sequences [2, 10].

$P f_{-}$and $P v_{-} 18 \mathrm{~S}$ rRNA qPCR was performed at all sites with the same protocols [2]. The same applies for $P f_{-}$varATS and $P v_{-}$mtCOX1 us-qPCR assays $[10,11]$, the performance of which is summarized in Table 1. For a comparison of parasite densities between study sites, qPCR efficiency and sensitivity of each assay was analysed across laboratories (Additional file 1: Table S1, Additional file 2: Fig. S1). Except for 5 Brazilian samples that were initially positive by $P f \_18 \mathrm{~S}$ rRNA qPCR, sufficient DNA for us-qPCR was available for all samples. In the comparative analysis of overall prevalence by varATS

Table 1 Molecular diagnostic assays used in this study

\begin{tabular}{|c|c|c|c|c|c|}
\hline Molecular assays & Target gene & $\begin{array}{l}\text { Amplicon length } \\
\text { (bp) }\end{array}$ & $\begin{array}{l}\text { Target copies/parasite } \\
\text { genome }\end{array}$ & LOD $^{\mathrm{a}}$ (parasite/ $\mu \mathrm{L}$ ) & References \\
\hline \multicolumn{6}{|l|}{ P. falciparum qPCR } \\
\hline Pf_18S rRNA & 18S rRNA genes & 221 & 3 & 1.57 & [2] \\
\hline Pf_varATS & var genes & 65 & $\geq 59$ & $0.06-0.15$ & [11] \\
\hline \multicolumn{6}{|l|}{ P. vivax $\mathrm{qPCR}$} \\
\hline PV_18S rRNA & 18S rRNA gene & 221 & 1 & $0.13-0.92$ & {$[2,12]$} \\
\hline Pv_mtCOX1 & $m+\mathrm{COX}_{1}$ & 148 & $>20$ & $0.01-0.023$ & {$[10,12]$} \\
\hline
\end{tabular}

a LOD for Pf_varATS qPCR determined by a serial dilution of the WHO International Standard (presented as parasites/ $\mu \mathrm{L}$ whole blood, reconstituted using WHO international standard together with uninfected blood); LOD for $P v \_m t C O X 1$ determined in two trendlines generated from LM-quantified field samples (presented as parasites $/ \mu \mathrm{L}$ whole blood from field samples) 
qPCR across the three sites, these 5 Brazilian samples were included and considered positive, justified by their original $P f \_18 \mathrm{~S}$ rRNA qPCR positivity and by data from Thailand and PNG, where 100\% (Thailand) or 98\% of 18S rRNA-positives (PNG) were also positive by varATS qPCR (Additional file 2: Fig. S2). In the analysis of parasite densities, the quantification of these 5 samples were based on the earlier $P f_{-} 18 \mathrm{~S}$ rRNA qPCR data.

\section{Data analysis}

Data were analysed using R.Studio version 3.5.1. Parasite densities determined by standard qPCR and us-qPCR assays were $\log 10$ transformed and presented as median densities, if not stated otherwise. A febrile malaria case was defined as an individual with fever or history of fever (body temperature $\geq 37.5{ }^{\circ} \mathrm{C}$ at blood sampling or within the preceding 2 days) and microscopic detection of parasites. Proportions of samples positive by standard 18S rRNA qPCR and us-qPCR were compared using McNemar's $\mathrm{Chi}^{2}$ test. A linear regression model was used to evaluate the difference of proportional gains by us-qPCR among the 3 study sites.

\section{Results}

Plasmodium falciparum-positivity by qPCR and us-qPCR

By standard $P f_{-}$18S rRNA qPCR, positivity was significantly higher in PNG compared to the other field sites $\left(\mathrm{Chi}^{2}\right.$-test, $\left.\mathrm{p}<0.001\right)$. The absolute number of additionally detected $P$. falciparum infections by us-qPCR was highest in PNG, the site with intermediate transmission intensity. The relative proportion of additionally detected P. falciparum infections differed substantially between Brazil (55\%, 95\% CI 25-82) and Thailand (33\%, 95\% CI 13-61) and PNG (30\%, 95\% CI 21-40) (Table 2, Fig. 1a, c), but owing to the small number of $P$. falciparum samples at the sites of low endemicity, these differences in gains were not statistically significant $(p=0.77)$.

\section{Plasmodium vivax prevalence by $\mathrm{qPCR}$ and us-qPCR} Plasmodium vivax was the prevailing species in Thailand and Brazil with 3.9\% (30/773) and 4.9\% (32/651) $P v \_18$ S rRNA-positive individuals. $P v_{-} 18 \mathrm{~S}$ rRNA positivity at these field sites was significantly lower than in PNG $\left(8.0 \%, 66 / 828, \mathrm{Chi}^{2}\right.$-test, $\left.\mathrm{p}<0.001\right)$ (Table 2), where $P$. vivax and $P$. falciparum prevalence rates were comparable (Table 2). The absolute number of $P$. vivax infections that were additionally detected by $P v_{-}$mtCOX1 us-qPCR was highest in PNG (Table 2), which in global comparison represents particularly high $P$. vivax prevalence. The proportion of $P$. vivax infection additionally detected by us-qPCR was similar in low endemic Thailand (23\%, 95\% CI 12-40) and Brazil (24\%, 95\% CI 13-40), but was higher in PNG (31\%, 95\% CI 22-41) (Fig. 1b). By usqPCR P. falciparum/P. vivax co-infections were detected in $4.1 \%(34 / 828)$ individuals from PNG and in 0.6\% (5/773) individuals from Thailand, whereas no co-infection was observed in Brazil.

\section{Plasmodium falciparum and $P$. vivax densities}

Quantification by us-qPCR was reliable as shown by a good correlation with quantification by $18 \mathrm{~S}$ rRNA qPCR (Additional file 2: Fig. S3). Characteristics of both us-qPCR assays that are relevant for quantification, agreed well between the different laboratories (Additional file 1: Table S1, Additional file 2: Fig. S1), hence, between-site comparisons of parasite densities by us-qPCR are appropriate. In contrast, densities of $P$. falciparum and $P$. vivax by us-qPCR cannot be compared directly for several reasons: Firstly, both multicopy assays target different numbers of gene copies

Table $2 P$. falciparum and $P$. vivax positivity in community samples from Thailand, Brazil and PNG, determined by speciesspecific 18S rRNA (qPCR) versus ultra-sensitive qPCR (us-qPCR)

\begin{tabular}{|c|c|c|c|c|c|c|}
\hline & \multicolumn{3}{|c|}{ P. falciparum positivity (\%) } & \multicolumn{3}{|c|}{ P. vivax positivity (\%) } \\
\hline & $\begin{array}{l}\text { qPCR } \\
(95 \% \mathrm{Cl}) \\
(\mathrm{n} / \mathrm{N})\end{array}$ & $\begin{array}{l}\text { us-qPCR } \\
(95 \% \mathrm{Cl}) \\
(\mathrm{n} / \mathrm{N})\end{array}$ & $p$-value ${ }^{a}$ & $\begin{array}{l}\text { qPCR } \\
(95 \% \mathrm{Cl}) \\
(\mathrm{n} / \mathrm{N})\end{array}$ & $\begin{array}{l}\text { us-qPCR } \\
(95 \% \mathrm{Cl}) \\
(\mathrm{n} / \mathrm{N})\end{array}$ & $p$-value ${ }^{\mathrm{a}}$ \\
\hline Thailand & $\begin{array}{l}1.3 \% \\
(0.7-2.5) \\
(10 / 773)\end{array}$ & $\begin{array}{l}1.9 \% \\
(1.1-3.3) \\
(15 / 773)\end{array}$ & 0.074 & $\begin{array}{l}3.90 \% \\
(2.7-5.6) \\
(30 / 773)\end{array}$ & $\begin{array}{l}5.00 \% \\
(3.7-6.9) \\
(39 / 773)\end{array}$ & 0.008 \\
\hline Brazil & $\begin{array}{l}0.80 \% \\
(0.3-1.9) \\
(5 / 651)\end{array}$ & $\begin{array}{l}1.70 \% \\
(0.9-3.1) \\
(11 / 651) \\
(11 / 651)\end{array}$ & 0.041 & $\begin{array}{l}4.90 \% \\
(3.4-6.9) \\
(32 / 651)\end{array}$ & $\begin{array}{l}6.50 \% \\
(4.7-8.7) \\
(42 / 651)\end{array}$ & 0.004 \\
\hline PNG & $\begin{array}{l}8.60 \% \\
(6.8-10.7) \\
(71 / 828)\end{array}$ & $\begin{array}{l}12.20 \% \\
(10.1-14.7) \\
(101 / 828)\end{array}$ & $<0.001$ & $\begin{array}{l}8.00 \% \\
(6.3-10.1) \\
(66 / 828)\end{array}$ & $\begin{array}{l}11.50 \% \\
(9.4-13.8) \\
(95 / 828)\end{array}$ & $<0.001$ \\
\hline
\end{tabular}

\footnotetext{
a McNemar's Chi ${ }^{2}$-test used to determine if gain of infections by us-qPCR was significant
} 

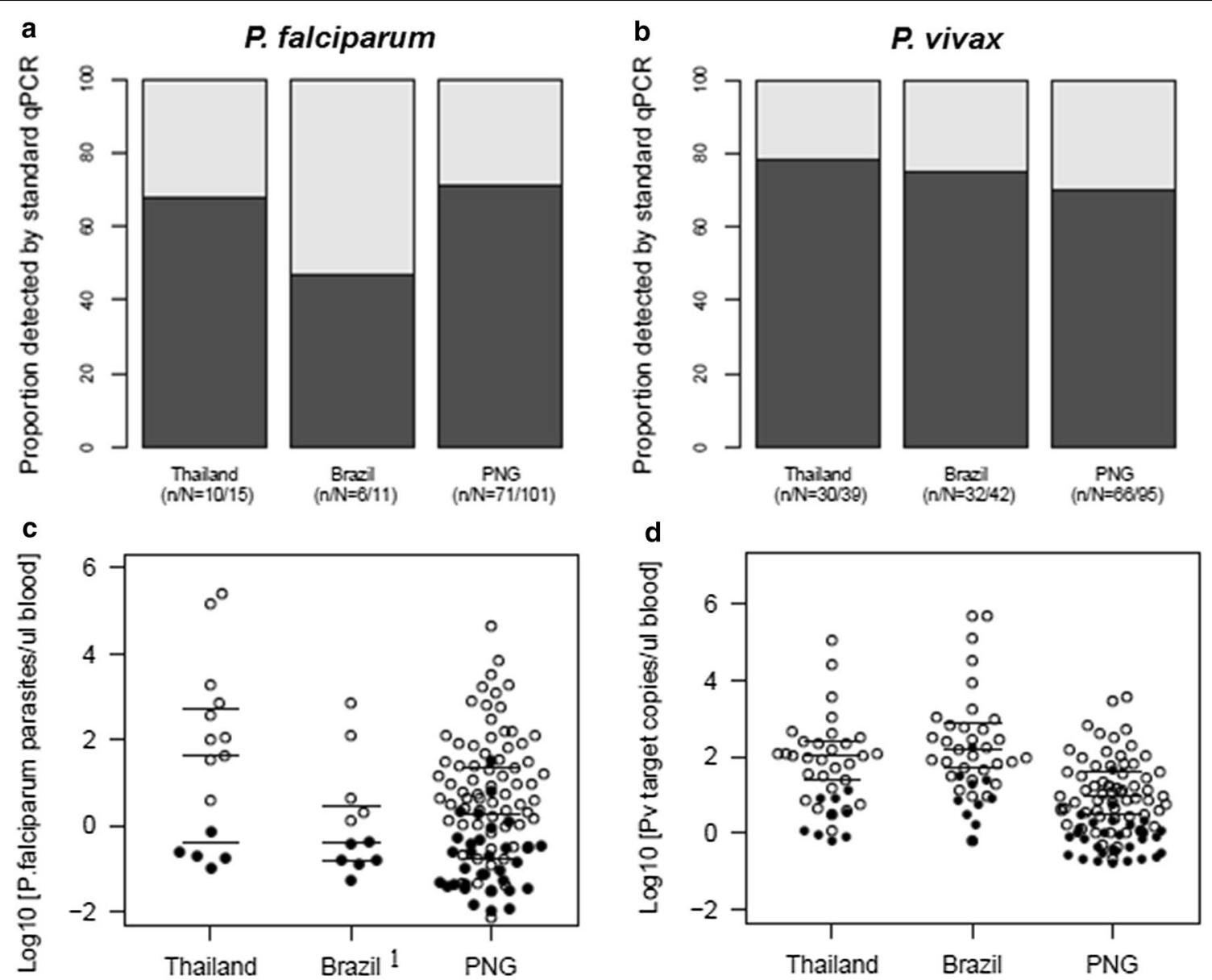

Fig. 1 Proportion of $P$. falciparum and $P$. vivax infections detected by qPCR versus us-qPCR by country. Proportion of $P$. falciparum (a) and $P$. vivax infections (b) positive by species-specific 185 rRNA qPCR among all samples positive by any qPCR assay (standard qPCR or us-qPCR) and parasite densities of $P$. falciparum (c) and P. vivax infections (d) detected by $18 \mathrm{~S}$ rRNA qPCR versus only by us-qPCR. White circles: infection detected by standard qPCR; black circles: infection detected only by us-qPCR. ${ }^{1}$ Owing to exhaustion of DNA, the parasite densities of 5 Brazilian samples (white circles) were quantified by 185 rRNA qPCR

per genome, thus, a comparison based on numbers of detected gene copies/ $\mu \mathrm{L}$ would be disproportionate. Secondly, P. falciparum densities were reported as parasites/ $\mu \mathrm{L}$ blood by converting $\mathrm{Ct}$-values determined by $P f_{-}$varATS us-qPCR into parasite numbers using a parasite trendline, whereas for $P$. vivax, owing to the lack of in vitro culture, a well-defined $P$. vivax parasite trendline was not available for absolute parasite quantification. Instead, $P$. vivax densities were derived from a dilution series of a plasmid standard and reported as $P v_{-}$mtCOX1 copy numbers/ $\mu \mathrm{L}$ blood. While densities of $P$. falciparum and $P$. vivax are not comparable between species, the median densities of either species are well comparable between field sites. For all countries, $P$. falciparum and $P$. vivax densities by us-qPCR were stratified according to a sample's positivity by either of the qPCR assays or both assays. (Table 3 and Fig. 1c, d).

The median parasite density of $P$. falciparum infections detected by $P f$ _ 18 S rRNA qPCR was highest in low-endemic Thailand, but significantly lower in lowendemic Brazil and moderate-endemic PNG (Table 3). This difference in median density may be attributed to symptomatic cases in the Thai sample set. When all symptomatic $P$. falciparum infections with densities $>1000$ parasites/ $\mu \mathrm{L}(3 / 15)$ were excluded from the analysis of Thai community samples, the median $P$. falciparum density decreased from 238.9 parasites/ $\mu \mathrm{L}$ to 11.2 parasites $/ \mu \mathrm{L}$ (IQR: $0.2-102.3$ parasites $/ \mu \mathrm{L}$ ). These densities from asymptomatic Thai study participants are in the same range as densities observed in PNG and Brazil (Fig. 1c). In all three settings, parasite densities in infections detected only by us-qPCR were 10-20 times 
Table 3 P. falciparum and $P$. vivax densities stratified according to positivity by standard 18S rRNA qPCR (qPCR), by any qPCR, or only by ultra- sensitive qPCR (us-qPCR)

\begin{tabular}{|c|c|c|c|c|c|c|}
\hline \multirow[t]{2}{*}{ Country } & \multicolumn{3}{|c|}{$\begin{array}{l}\text { P. falciparum Median Density } \\
\text { Parasites/uL (IQR) }\end{array}$} & \multicolumn{3}{|c|}{$\begin{array}{l}\text { P. vivax Median Density }{ }^{\mathrm{a}} \\
\text { Pv_mtCOX1 copies/ } \mu \mathrm{L} \text { (IQR) }\end{array}$} \\
\hline & $\mathrm{qPCR}(+)$ & $\begin{array}{l}\mathrm{qPCR}(+) \\
\text { and us- } \mathrm{qPCR}(+)\end{array}$ & $\begin{array}{l}\text { qPCR(-) } \\
\text { and us-qPCR(+) }\end{array}$ & $\mathrm{qPCR}(+)$ & $\begin{array}{l}\mathrm{qPCR}(+) \\
\text { and us-qPCR( }(+)\end{array}$ & qPCR(-) and us-qPCR(+) \\
\hline Thailand & $238.9(55.8-1519)$ & $40.8(0.5-536.2)$ & $0.2(0.2-0.2)$ & $104.8(24.9-246.7)$ & $50.4(4.9-190.8)$ & $3.1(0.9-7.6)$ \\
\hline Brazil & $4.0^{\mathrm{b}}(1.9-125.4)$ & $0.4^{\mathrm{b}}(0.2-3.0)$ & $0.2(0.1-0.3)$ & $152.6(50.3-738.7)$ & $79.8(18.3-464.4)$ & $7.3(3.7-22.0)$ \\
\hline PNG & $5.7(1.0-70.0)$ & $1.9(0.2-22.5)$ & $0.1(0.0-0.4)$ & $9.5(3.1-145.9)$ & $3.7(0.9-16.4)$ & $0.7(0.3-1.2)$ \\
\hline
\end{tabular}

${ }^{a} \log _{10}$ transformed median parasite density quantified by us-qPCR

b Owing to exhaustion of DNA, parasite densities of 5 of the Brazilian samples were quantified by $18 \mathrm{~S}$ rRNA qPCR

lower than densities in infections only positive by the less sensitive $P f_{-} 18 \mathrm{~S}$ rRNA qPCR $(\mathrm{p}<0.001)$ (Table 3).

Just as for P. falciparum, P. vivax parasite densities in infections only detected by us-qPCR were 8-10 times lower than densities in $P$. vivax infections detected by $P v \_18$ S rRNA qPCR $(\mathrm{p}<0.001)$ (Table 3, Fig. 1d). The median $P$. vivax parasite density in $P$. vivax-positives detected by $P v_{-} 18 \mathrm{~S}$ rRNA qPCR was 11 -fold lower in community samples from PNG than in those from Thailand, and 16 -fold lower than in Brazil $(\mathrm{p}<0.05)$ (Table 3). Similarly, the median $P$. vivax density in samples only positive by us-qPCR was significantly lower in PNG compared to Thailand and Brazil $(\mathrm{p}<0.05)$ (Table 3).

\section{Age trends of $P$. falciparum and $P$. vivax infections}

To identify population sub-groups that harbour particularly low-density infections and where benefits from usqPCR would thus be greatest, parasite prevalence and density was analysed with respect to age. In all study sites, $P$. vivax positivity varied by age. This trend was consistent by both diagnostic assays. The age-stratified prevalence rates generated by the two molecular assays did not differ significantly at any study site $\left(\mathrm{Chi}^{2}\right.$; Thailand:
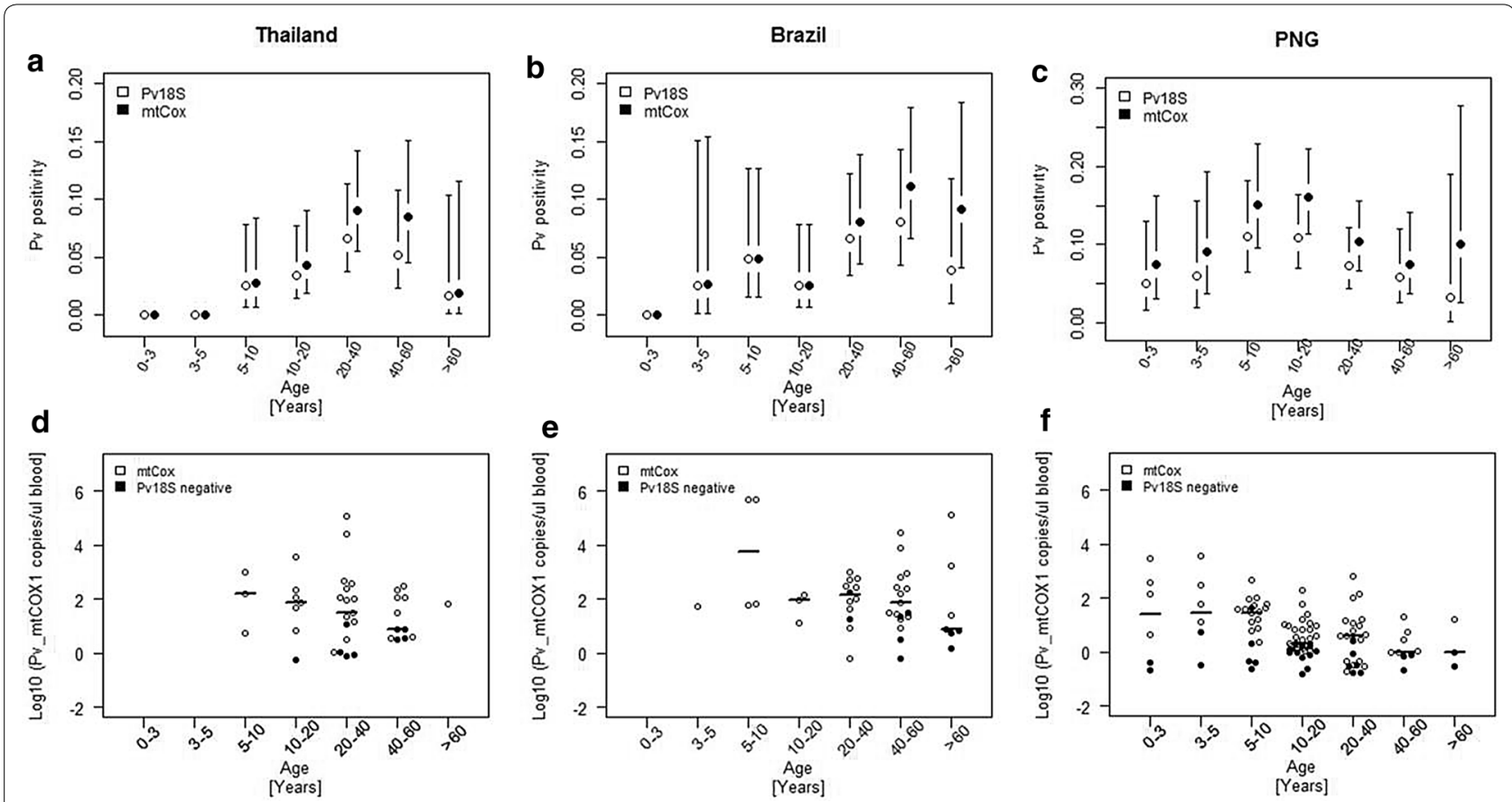

Fig. 2 Age trends in P. vivax positivity and parasite density in Thailand, Brazil and PNG. P. vivax infections detected by Pv_18S rRNA qPCR and $P v \_$mtCOX 1 us-qPCR (white circles) and $P$. vivax infections only detected by $P v_{-}$mtCOX 1 us-qPCR (black circles). a-c $P$. vivax positivity by age group with $95 \%$ confidence intervals (black vertical lines). $\mathbf{d}-\mathbf{f} \log _{10}$ transformed $P$. vivax parasite density by $P v \_m t C O X 1$ us-qPCR per age group with median Pv_mtCOX1 copy numbers (horizontal lines) 
$\mathrm{p}$-value $=0.96$, Brazil: $\mathrm{p}=0.79$, PNG: $\mathrm{p}=0.73)$. In Thailand and Brazil, $P$. vivax prevalence by both, standard and us-qPCR, peaked in adults aged 20-60 years (Fig. 2a, b), whereas in PNG prevalence was highest by both assays in adolescents aged 10-20 years (Fig. 2c). No P. vivax infection was found in the youngest age group in Brazil and Thailand ( $0-3$ years), whereas $P$. vivax infections were detected by both assays in children from PNG aged 0-3 years (Fig. 2a-f). Plasmodium vivax infections were also absent in Thai children aged 3-5 years. At all three sites, median $P$. vivax densities measured by $P v_{-} \mathrm{mtCox} 1$ copies $/ \mu \mathrm{L}$ blood were highest in the youngest age groups that included $P$. vivax-positive individuals, and tended to decrease by age (Fig. $2 \mathrm{~d}-\mathrm{f}$ ). These trends were not statistically significant owing to the limited number of $P$. vivax-positive individuals in some age groups.

Age trends for P. falciparum prevalence and parasite density in Thailand and Brazil could not be analysed because of the limited number of $P$. falciparum-positive individuals. For PNG, the age distribution of $P$. falciparum infections is shown in Additional file 2: Fig. S4.

\section{Discussion}

This study investigated the extent of low-density $P$. falciparum and $P$. vivax infections in moderate and low transmission settings in Thailand, Brazil and PNG, using ultra-sensitive detection assays. The LODs of the $P$. falciparum and $P$. vivax us-qPCR assays, which was 10 -times lower than the standard assay $P f_{-} 18$ S rRNA qPCR [10, 11 ] were in good agreement between the three laboratories. The inter-laboratory comparability of results was closely controlled by the use of external reference standards. To evaluate the usefulness of us-qPCR assays for epidemiological studies, age trends in prevalence rates were analysed. No differences were observed in agedependent epidemiological pattern in prevalence nor density in any transmission setting, thus confirming the suitability of us-qPCR for epidemiological research.

Substantial differences in gains were observed across transmission settings. For example, at the PNG study site, $P$. falciparum prevalence in the community increased from $8.6 \%$ to $12.2 \%$ by us-qPCR, an increase of $30 \%$ in $P$. falciparum positivity. In Brazil P. falciparum prevalence was much lower and a two-fold gain was observed in the proportion of positive samples by use of the ultra-sensitive varATS assay. However, the absolute numbers of $P$. falciparum infections in this comparative study were too low (i.e. a rise from 6/651 to 11/651 P. falciparumpositive individuals) so that the difference between study sites in the proportional gains was not statistically significant. The question whether proportional gains are higher at very low $P$. falciparum transmission intensity remains to be clarified by a larger study with a sufficient $P$. falciparum sample size for statistical analysis. Gains that differ by transmission intensity could occur if low-density $P$. falciparum infections undetectable by standard $P f_{-} 18 \mathrm{~S}$ rRNA qPCR, would be relatively more abundant in low compared to high transmission. Earlier meta-analyses of global prevalence data of both, PCR- and microscopybased diagnosis $[1,16]$ had revealed a negative relationship of the proportion of submicroscopic infections with transmission intensity.

The seemingly lower proportional gain in PNG compared to the Brazilian site (30\% versus $55 \%$ ) might be related to slightly higher overall $P$. falciparum parasite densities in community samples from PNG (1.9 parasites $/ \mu \mathrm{L})$ compared to Brazil $(0.4$ parasites $/ \mu \mathrm{L})$. The small difference in median $P$. falciparum densities between PNG and Brazil is in proximity to the LOD of the $P f_{-} 18 \mathrm{~S}$ rRNA assay (1.57 parasites/ $\mu \mathrm{L}$ blood) and therefore could have caused a higher proportion of infections in PNG samples to be detectable by standard $P f_{-} 18 \mathrm{~S}$ rRNA qPCR. Whether density differences between sites could explain the smaller gains in PNG by using us-qPCR remains unclear, as the limited number of $P$. falciparum cases from low endemic Brazil precludes clarification of reasons for differing proportional gains.

Among all three sites, the P. falciparum median density was highest in Thailand with 238 parasites $/ \mu \mathrm{L}$ compared to 4.0 parasites $/ \mu \mathrm{L}$ in Brazil. This was due to very few high density infections with $>1000$ parasites $/ \mu \mathrm{L}$ in this community. In addition, 3 of these $P$. falciparuminfected cases showed evidence of fever within the preceding 2 days. These findings from Thailand could mirror declining transmission and indicate that less frequent exposure to $P$. falciparum may result in waning immunity against this parasite. This would suggest that $P$. falciparum-infected individuals at the Thai study site more often develop high parasite densities and become symptomatic and thus will receive treatment. It, therefore, can be speculated that the presence of malaria episodes has caused a lower proportional gain of infections detected by usqPCR in Thailand compared to low-endemic Brazil.

$P v \_$mtCOX us-qPCR resulted in an analogical increase in the proportion of additionally detected $P$. vivax-infections across all study sites, ranging from $31 \%$ in PNG to $24 \%$ in Brazil and $23 \%$ in Thailand. Compared to the more pronounced differences in P. falciparum proportional gains, the $P v_{-} \mathrm{mtCOX}$-based increase was similar across the 3 sites. When $P$. vivax median densities were consulted to explain the small difference in proportional gains between study sites, 11-16-fold higher $P$. vivax densities were observed in Brazil and Thailand compared to PNG. This might be responsible for the lower gain by more sensitive diagnostics in these two low endemic settings. 
Benefits from us-qPCR consist in the improved detection of low-density $P$. falciparum and $P$. vivax infections. The question arises, whether these low-density infections carry gametocytes in sufficient numbers to contribute to onward transmission to mosquitoes. This question has been addressed by previous studies at all three sites, either by membrane feeding assays on symptomatic blood samples $[20,21]$ or by using gametocyte density as surrogate for transmission potential, such as in a study recently performed in the same community in PNG, where gametocytes were quantified after enrichment from large volumes of blood [12]. In this recent study, more than half of the P. falciparum- and $P$. vivax-positive samples that were only detected by us-qPCR, carried gametocytes, (10 gametocyte carriers in 15 P. falciparum-infections and 11 gametocyte carriers in $19 P$. vivaxinfections) [12]. Gametocyte densities were tenfold lower in infections detected by standard $18 \mathrm{~S}$ rRNA qPCR compared to infections only detectable by us-qPCR and were below 1 male and 1 female gametocyte/ $\mu \mathrm{L}$ [22]. Although the gametocyte sex-ratio suggested a very low likelihood of transmission [22], it cannot be excluded that parasite densities in untreated, chronic malaria infections in the community may fluctuate and transiently reach levels that could potentially be infective to mosquitoes [23].

A limitation in the quantification of $P$. vivax infections derives from differences in the numbers of mitochondria between different $P$. vivax blood stages, i.e. between a ring stage versus a multi-nucleated schizont stage, both present in peripheral blood. The number of target gene copies per parasite varies accordingly [10] [17]. Plasmodium vivax parasite densities calculated from qPCR copy numbers thus represent only an estimate. Yet, this equally applies to the variable number of nuclear genomes and thus to $18 \mathrm{~S}$ rDNA copies circulating in $P$. vivax parasites of any stage. This explains our observation of a good correlation between both markers for quantification of $P$. vivax. For other human Plasmodium species occurring at the 3 study sites, us-qPCR assays have not yet been developed, thus the analyses performed in this study were limited to $P$. falciparum and $P$. vivax.

Investigations of individuals at greatest risk of infection based on parasite detection by $P f_{-}$and $P v_{-} 18 \mathrm{~S}$ rRNA qPCR assays have been described previously for all three study sites [19, 24] [W. Monteiro, personal communication]. These earlier analyses showed that main predictors of $P$. falciparum and $P$. vivax infections and density differed substantially between sites. To complement these former epidemiological analyses, age trends of $P$. falciparum and $P$. vivax infection in relation to parasite densities based on us-qPCR data were investigated. Parasite carriers with very low-density infections in PNG were primarily found in adolescents aged $10-20$ years, whereas in
Thailand and Brazil most additionally gained infections were found in older individuals ( $>40$ years of age). Therefore, the additional $P$. vivax infections detected by usqPCR coincided with the age group that had presented the highest risk of infection in earlier analyses from Thailand, Brazil and PNG [19, 24] (W.M. Monteiro, pers. commun.).

Depending on the public health question to be addressed by molecular diagnosis, more precise prevalence data as achieved by us-qPCR may be of considerable relevance, particularly in low transmission settings, where each individual malaria case is investigated by elimination programmes [25]. Furthermore, results are likely to be more consistent across surveys, as stochastic amplification of low-density infections is reduced $[2,11]$. Because this study demonstrated a substantial gain of additionally detected low-density infections, particularly in low transmission settings, us-qPCR could be crucial for surveillance in elimination settings. Molecular diagnosis is increasingly used to determine presence of infections to guide control efforts [26]. The use of us-qPCR, and thus greater confidence in diagnostic metrics, could reduce the number of samples to be screened before declaring a region malaria-free.

\section{Conclusion}

The use of us-qPCR for parasite detection in the community provided a more accurate picture of low-density infections at different levels of transmission intensity. The benefits of using ultra-sensitive assays were clearly demonstrated for all study sites. When analysing the relationship of low versus high transmission intensity and the gain in $P$. vivax positivity by us-qPCR, only minor differences were observed between study sites in the $P$. vivax proportional gains in positivity that ranged from 23\% to $31 \%$. The P. falciparum proportional gain was almost twice as high in Brazil (low P. falciparum transmission) than in PNG (high P. falciparum transmission). However, this difference was not statistically significant, as the absolute number of $P$. falciparum positive samples in the Brazilian community survey was as low as $1.7 \%$ $(11 / 651)$ by $P$. falciparum us-qPCR. Further comparative studies are required to clarify a potentially higher gain in $P$. falciparum positivity in low transmission versus high transmission.

In areas of low P. falciparum and $P$. vivax transmission, the absolute number of infections additionally gained by ultra-sensitive methods seem few. However, for certain research questions arising in pre-elimination settings, such increased sensitivity might be beneficial for informing control measures. For instance, $P v_{-}$us-qPCR can play an important role in both epidemiological studies and surveillance, because those additionally detected 
$P v$-positive individuals may experience relapses and in future contribute to onwards transmission.

\section{Supplementary information}

Supplementary information accompanies this paper at https://doi. org/10.1186/s12936-020-03374-7.

Additional file 1. Supplementary Table.

Additional file 2. Supplementary Figures.

\section{Abbreviations}

DNA: Deoxyribonucleic acid; LM: Light microscopy; LOD: Limit of detection; mtCOX1: Mitochondrial cytochrome C oxidase 1; PCR: Polymerase chain reaction; PNG: Papua New Guinea; qPCR: Quantitative polymerase chain reaction; RDT: Rapid diagnostic test; us: Ultra-sensitive; varATS: Var gene acidic terminal sequence.

\section{Acknowledgements}

We thank all study participants as well as field and laboratory teams at the three study sites.

\section{Authors' contributions}

MG, CAM, NEH, EN performed laboratory work and analysed data. NEH, CK, LJR, WMM, GM, AK, AMS, WN, QB, IM helped to edit the manuscript. IF, IM, JS, $M L$ and AMS contributed to conception, study design and supervision of field projects. MG and IF wrote the manuscript. All authors read and approved the final manuscript.

\section{Funding}

This study was funded by the Swiss National Science Foundation (grants no. 310030_159580 and IZRJZ3_164182), the TransEPi Consortium/Bill and Melinda Gates Foundation (OPP1034577) and by the NIH (1 U19 AI089686-01). IM was supported by an NHMRC Senior Research Fellowship.

\section{Availability of data and materials}

All data presented in this article or accompanying Additional files can be made available upon request.

\section{Ethics approval and consent to participate}

Scientific approval and ethical clearance for the PNG cross-sectional study was obtained from the PNG Institute of Medical Research Institutional Review Board (IMR IRB) (1116/1204), and the PNG Medical Research Advisory Committee (MRAC) (11.21/1206). Additional clearance for the re-analyses was obtained from the Medical Research Advisory Committee of the PNG Government, MRAC no. 16-01. Approval for the field study in Thailand was obtained from the Ethics Committee of the Faculty of Tropical Medicine, Mahidol University, Thailand (EC approval number MUTM 2012-044-01). The Brazilian study was approved by the Brazilian National Committee of Ethics (CONEP) (349.211/2013). Informed written consent was obtained from all participants or their legal guardians prior to sampling. Ethical approval for diagnostic evaluations using archived samples was obtained in Switzerland from Ethikkommission Nordwest- und Zentralschweiz, EKNZ Req-2016-000050.

\section{Consent for publication}

All authors declare their consent to publish the manuscript and agree to the proposed authorship order.

\section{Competing interests}

The authors declare that they have no competing interests.

\section{Author details}

1 Swiss Tropical and Public Health Institute, Basel, Switzerland. ${ }^{2}$ University of Basel, Basel, Switzerland. ${ }^{3}$ Walter and Eliza Hall Institute of Medical Research, Melbourne, Australia. ${ }^{4}$ Papua New Guinea Institute of Medical Research, Madang, Papua New Guinea. ${ }^{5}$ Fundação de Medicina Tropical Dr. Heitor Vieira Dourado (FMT-HVD), Manaus, Brazil. ${ }^{6}$ Instituto Nacional de Infectologia Evandro Chagas, Fiocruz, Rio de Janeiro, Brazil. ${ }^{7}$ Department of Molecular
Tropical Medicine \& Genetics, Faculty of Tropical Medicine, Mahidol University, Bangkok, Thailand. ${ }^{8}$ ISGlobal, Hospital Clínic - Universitat de Barcelona, BarceIona, Spain. ${ }^{9}$ Universidade Do Estado Do Amazonas, Manaus, Brazil. ${ }^{10}$ Mahidol Vivax Research Unit, Faculty of Tropical Medicine, Mahidol University, Bangkok, Thailand. ${ }^{11}$ Department of Medical Biology, University of Melbourne, Melbourne, Australia. ${ }^{12}$ Present Address: Eck Institute for Global Health, University of Notre Dame, Notre Dame, IN, USA. ${ }^{13}$ Present Address: Burnet Institute, Melbourne, Australia. ${ }^{14}$ Present Address: Malaria Parasite \& Hosts Unit, Institut Pasteur, Paris, France.

Received: 28 April 2020 Accepted: 13 August 2020

Published online: 03 September 2020

\section{References}

1. Okell LC, Bousema T, Griffin JT, Ouédraogo AL, Ghani AC, Drakeley CJ. Factors determining the occurrence of submicroscopic malaria infections and their relevance for control. Nat Commun. 2012;3:1237.

2. Wampfler R, Mwingira F, Javati S, Robinson L, Betuela I, Siba P, et al. Strategies for detection of Plasmodium species gametocytes. PLoS ONE. 2013;8:e76316.

3. Koepfli C, Robinson LJ, Rarau P, Salib M, Sambale N, Wampfler R, et al. Blood-stage parasitaemia and age determine Plasmodiumfalciparum and P. vivax gametocytaemia in Papua New Guinea. PLoS ONE. 2015;10:e0126747.

4. Almeida ACG, Kuehn A, Castro AJM, Vitor-Silva S, Figueiredo EFG, Brasil LW, et al. High proportions of asymptomatic and submicroscopic Plasmodium vivax infections in a peri-urban area of low transmission in the Brazilian Amazon. Parasit Vectors. 2018;11:194.

5. Nguitragool W, Mueller I, Kumpitak C, Saeseu T, Bantuchai S, Yorsaeng R, et al. Very high carriage of gametocytes in asymptomatic low-density Plasmodium falciparum and P. vivax infections in western Thailand. Parasit Vectors. 2017; 10:512.

6. Imwong M, Nguyen TN, Tripura R, Peto TJ, Lee SJ, Lwin KM, et al. The epidemiology of subclinical malaria infections in South-East Asia: findings from cross-sectional surveys in Thailand-Myanmar border areas, Cambodia, and Vietnam. Malar J. 2015;14:381.

7. Kamau E, Alemayehu S, Feghali KC, Saunders D, Ockenhouse CF. Multiplex qPCR for detection and absolute quantification of malaria. PLoS ONE. 2013;8:e71539.

8. Shokoples SE, Ndao M, Kowalewska-Grochowska K, Yanow SK. Multiplexed real-time PCR assay for discrimination of Plasmodium species with improved sensitivity for mixed infections. J Clin Microbiol. 2009;47:975-80.

9. Rosanas-Urgell A, Mueller D, Betuela I, Barnadas C, Iga J, Zimmerman PA, et al. Comparison of diagnostic methods for the detection and quantification of the four sympatric Plasmodium species in field samples from Papua New Guinea. Malar J. 2010;9:361.

10. Gruenberg M, Moniz CA, Hofmann NE, Wampfler R, Koepfli C, Mueller I, et al. Plasmodium vivax molecular diagnostics in community surveys: pitfalls and solutions. Malar J. 2018;17:55.

11. Hofmann N, Mwingira F, Shekalaghe S, Robinson LJ, Mueller I, Felger I. Ultra-sensitive detection of Plasmodium falciparum by amplification of multi-copy subtelomeric targets. PLoS Med. 2015;12:e1001788.

12. Hofmann NE, Gruenberg M, Nate E, Ura A, Rodriguez-Rodriguez D, Salib $M$, et al. Assessment of ultra-sensitive malaria diagnosis versus standard molecular diagnostics for malaria elimination: an in-depth molecular community cross-sectional study. Lancet Infect Dis. 2018;18:1108-16.

13. Imwong M, Stepniewska K, Tripura R, Peto TJ, Lwin KM, Vihokhern B, et al. Numerical distributions of parasite densities during asymptomatic malaria. J Infect Dis. 2016;213:1322-9.

14. Demas A, Oberstaller J, DeBarry J, Lucchi N, Srinivasamoorthy G, Sumari $D$, et al. Applied genomics: data mining reveals species-specific malaria diagnostic targets more sensitive than 18S rRNA. J Clin Microbiol. 2011;49:2411-8.

15. Lucchi NW, Poorak M, Oberstaller J, DeBarry J, Srinivasamoorthy G, Goldman I, et al. A new single-step PCR assay for the detection of the zoonotic malaria parasite Plasmodium knowlesi. PLoS ONE. 2012;7:e31848. 
16. Cheng Q, Cunningham J, Gatton ML. Systematic review of sub-microscopic P. vivax infections: prevalence and determining factors. PLoS Negl Trop Dis. 2015;9:e3413.

17. Preiser PR, Wilson RJ, Moore PW, McCready S, Hajibagheri MA, Blight KJ, et al. Recombination associated with replication of malarial mitochondrial DNA. EMBO J. 1996;15:684-93.

18. Moreira CM, Abo-Shehada M, Price RN, Drakeley CJ. A systematic review of sub-microscopic Plasmodium vivax infection. Malar J. 2015;14:360.

19. Koepfli C, Ome-Kaius M, Jally S, Malau E, Maripal S, Ginny J, et al. Sustained malaria control over an 8-year period in Papua New Guinea: the challenge of low-density asymptomatic Plasmodium infections. J Infect Dis. 2017;216:1434-43.

20. Martins-Campos KM, Kuehn A, Almeida A, Duarte APM, Sampaio VS, Rodriguez IC, et al. Infection of Anopheles aquasalis from symptomatic and asymptomatic Plasmodium vivax infections in Manaus, western Brazilian Amazon. Parasit Vectors. 2018;11:288.

21. Kiattibutr K, Roobsoong W, Sriwichai P, Saeseu T, Rachaphaew N, Suansomjit C, et al. Infectivity of symptomatic and asymptomatic Plasmodium vivax infections to a Southeast Asian vector Anopheles dirus. Int J Parasitol. 2017:47:163-70
22. Gruenberg M, Hofmann NE, Nate E, Karl S, Robinson LJ, Lanke K, et al. Molecular and immunofluorescence-based quantification of male and female gametocytes in low-density P. falciparum infections and their relevance for transmission. J Infect Dis. 2020;221:598-607.

23. Collins KA, Wang CYT, Adams M, Mitchell H, Rampton M, Elliott $S$, et al. A controlled human malaria infection model enabling evaluation of transmission-blocking interventions. J Clin Invest. 2018;128:1551-622.

24. Nguitragool W, Karl S, White M, Koepfli C, Felger I, Singhasivanon P, et al. Highly heterogeneous residual malaria risk in western Thailand. Int J Parasitol. 2019;49:455-62.

25. ZAMEP (2014) Zanzibar Guidelines for Malaria Diagnosis and Treatment Zanzibar Malaria Elimination Programme (ZAMEP).

26. Lemoine JF, Boncy J, Filler S, Kachur SP, Fitter D, Chang MA. Haiti's commitment to malaria elimination: progress in the face of challenges, 2010-2016. Am J Trop Med Hyg. 2017;97:43-8.

\section{Publisher's Note}

Springer Nature remains neutral with regard to jurisdictional claims in published maps and institutional affiliations.
Ready to submit your research? Choose BMC and benefit from:

- fast, convenient online submission

- thorough peer review by experienced researchers in your field

- rapid publication on acceptance

- support for research data, including large and complex data types

- gold Open Access which fosters wider collaboration and increased citations

- maximum visibility for your research: over 100M website views per year

At BMC, research is always in progress.

Learn more biomedcentral.com/submissions 